\title{
Ueber Drüsennerven.
}

Bericht iiber einige in Gemeinsehaft mit Th. W. van Lidth de $\mathrm{J} e u d \mathrm{e}$ angestellte Untersuchungen.

Von

Wh. W. Eggelmamn in Utrecht.

Die positiven Angaben, welche bisher über die Endigungsweise sekretorischer Nerven gemacht sind, erfreuen sich wie bekannt keineswegs allgemeiner Zustimmung. Die weitgreifenden Ergebnisse, zu denen Pflüger an den Speicheldrüsen und der Leber der Säugethiere gelangte, wurden und werden noch von allen Seiten her bestritten. Aber auch Kupffer's Mittheilungen über die Nervenendigungen in den Speicheldriisen von Periplaneta orientalis haben nicht den erwarteten Anklang gefunden. Sie erweckten die Hoffnung, dass hier ein Musterobjekt gefunden sei, welches nicht nur den wichtigsten Punkt, das Eindringen der Nervenfasern ins Innere der Drüsenbläschen bis zum Contakt mit den Zellen, sondern auch die besondere Weise der Verbindung von Nervenfibrillen und secernirender Zellsubstanz mit der handgreiflichsten Deutlichkeit zu erkennen und zu demonstriren gestatte.

Diese Hoffnung hat sich als trïgerisch erwiesen. Schon der Umstand musste im höchsten Grade verdächtig erscheinen, dass zur Demonstration der feinsten Fibrillen und ihrer Fortsetzungen im Drüsenprotoplasma Behandlung des frischen Objekts mit sehr verdünnter Kalilauge empfohlen wurde. Ist doch gerade dies Verfahren anerkanntermassen eines der allersichersten Mittel um Nervenfibrillen und zarte protoplasmatische Gebilde, gleichviel welcher Art, schnell bis zur völligen Unkenntlichkeit zu zerstören. Bei einer näheren Untersuchung des Kupffer'schen Objekts, die Herr van Lidth de Jeude unter meiner Mitwirkung anstellte, fanden wir denn auch, dass die weitaus grösste Zahl der angeblichen Nerven nicht wohl etwas anderes als Bindegewebsstränge sein können.

Es ist nämlich ganz sicher, dass viele dieser Stränge - ob- 
schon nicht alle - nirgends einen Zusammenhang mit unzweifelhaften Nerven aufweisen, auch nicht durch Vermittelung von Tracheen, sondern einfach, und ohne unterwegs Zweige abzugeben, entweder von einem Driisenläppchen zum anderen, oder von der Wand des grossen blasenförmigen Speichelbehälters, der neben jeder Speicheldrüse liegt, zu einem benachbarten Drüsenläppchen herüberziehen. Namentlich die letzteren Stränge eignen sich zur Feststellung dieses wichtigen Punltes. Wären sie wirklich Nerven, so würde man sie - ein bisher unerhörter Fall - als interperipherische, vom iubrigen Nervensystem des Körpers unabhängige Nerven zu betrachten haben. Obschon nan die Möglichkeit des Bestehens derartiger Nerven, wie mir scheint, a priori nicht geläugnet werden kann, so wird man doch nicht ohne die zwingendsten Gründe zu einer Annahme von so principieller Tragweite schreiten mögen.

Freilich zeigen nun die betreffenden Stränge in ihrem Bau so viel Aehnlichkeit mit echten Nervenstämmen, dass man sich nur ungern entschliesst, sie nicht für solche zu halten. Sie stellen nämlich, gerade wie diese, Röhren dar, mit fibrillärem Inhalt und Kernen in einer homogenen oder sehr zartkörnigen interfibrillären Masse. Die Fibrillen unterscheiden sich in Form, Dimensionen, optischen und chemischen Eigenschaften nicht merklich von den Nervenfibrillen der Verzweigungen des Bauchstrangs. Speciell sind sie wie diese einfach brechend, quellen und lösen sich, frisch mit kaustischen Alkalien behandelt, sehr sehnell auf, nehmen in $1 / 2 \%$ Osminmsäure nur schwachbräunliche, in Karmin deutliche Färbung an. Die Wand der Röhre, welche die Fibrillen umhüllt, stimmt mit der dicken Scheide echter Nervenstämme in der Regel völlig überein. Sie versehmilat mit der Membrana propria der Drisenbläschen und des Speichelbehälters, gerade wie die Nervenscheide beim Eintritt des Nerven in die Muskelfaser mit dem Sarkolemm. Der Inhalt der Stränge steht also mit dem Inhalt der Drüsenbläsehen, bezüglich des Speichelbehälters in offener Verbindung, und wirklich scheinen oft die Fibrillen wenigstens mit den Speicheldrüsenzellen in Contakt zu sein.

Es kommt nun aber auch vor, dass solche Stränge - wegen der beschriebenen Aehnlichkeiten mit Nervenfasern mögen sie Neuroidfasern heissen - gegen die Drise hin unter allmählichem Sehwinden des Lumens und der darin enthalte- 
nen Fibrillen sich hautartig abplatten und, verbreitert oder in chitinartige, sehr resistente Fasern zerspalten, an die Membrana propria eines Acinus sich ansetzen. Man findet auch derartige Fasern oder Faserbïndel, die in ihrem Verlauf von einem Läppehen zum andern nirgends eine röhrenförmige Beschaffenheit aufweisen.

Ganz ähnliehe Verhältnisse trifft man bei den Malpighischen Gefässen von Raupen. Die Neuroidfasern sind hier von Leydig ${ }^{1}$ ), $\mathrm{Chun}^{2}$ ) a. a. untersucht und als echte Drïsennerven gedeutet worden. Die histologische Uebereinstimmung mit Nerven geht hier sogar noch weiter als bei Periplaneta, indem die Röhren, wenigstens die dickeren, in ihrem Verlauf in der Regel eine oder mehrere spindelförmige ganglienartige Anschwellungen bilden. Wie in echten Ganglien findet man in diesen Anschwellnngen zwischen den Fibrillen eine grössere oder kleinere Zahl von rundlichen oder spindelförmigen Zellen eingelagert. Niemand, der diese Gebilde in Mikroskop sieht, wird daran zweifeln, dass er echte Nerven und Ganglien vor sich habe, wie ihnen denn auch bisher kaum jemals eine andere Deutung gegeben worden ist $\left.{ }^{3}\right)$. Und dennoch liefert die nähere Untersuchung gerade dieses Objektes die allergewichtigsten Einwände gegen eine solche Deutung.

Zunächst führt die Verfolgung der Fasern nach ihren Enden hin zu dem Ergebniss, dass dieselben nirgends in einen echten Nervenstamm einmünden, sondern rein interperipherische Verbindungen herstellen zwischen den Malpighisehen Gefässen einerseits und der Aussenwand des Darmes andererseits; und zwar verschmilzt am letzteren Orte ihre Membran entweder mit der zwischen Ringund Längsmaskelschicht des Darmkanals gelegenen Chitinhaut, oder setzt sich an eine quergestreifte Muskelfaser an, meist wie eine Sehne an das freie Ende eines Zweigs der grossen baumförmig verästelten Muskelfasern die sich anssen auf dem Raupendarm inseriren.

1) F. Leydig, Lehrb. d. Histologie etc. p. 474. 1857. - Derselbe, Bemerkungen über die Farbe der Fautdecken und Nerven der Drüsen bei Insekten. Arch. f. mikr. Anat. XII. p. 542. 1876.

2) Chun, Ueber den Bau, die Entwickelung and physiologische Bedeutung der Rektaldrüsen bei den Insekten. A. d. Abhandl. d. Senckenbergischen naturforsch. Gesellsch. in Frankfurt a. M. Bd. X. p. 23 flg. 1875.

3) Newport (Todd's Cyclopaed. of anat. etc. Art. Insecta) hielt sie, wie Chun schon bemerkt, für Gefässe.

E. Pflüger, Arohit f. Physiologie. Ba. XXrv. 
Die räumlichen Beziehungen des Inhalts der Neuroidröhren zum Muskelfaserinhalt sind derart, dass an eine wirklich nervöse physiologische Einwirkung des einen auf den andern nicht gedacht werden kann: die Fibrillen berühren die contraktile Substanz nicht. In vielen Fällen reicht der Inhalt der Neuroidröhre nur bis auf eine Entfernung von mehreren Hundertstelmillimetern an die Muskelfaser heran, wo dann die Fibrillen blind in der Röhre endigen (anch nicht umbiegen), und nur die Chitinscheide oder faserartige Fortsetzungen derselben bis zur Insertionsstelle reichen.

Ebensowenig gestatten unsere Beobachtungen, nervöse Beziehungen zu den Drüsenzellen der Malpighischen Gefässe anzunehmen. Entgegen den Angaben der obengenannten vortrefflichen Beobachter müssen wir nämlich behaupten, dass die Neuroidröhren nicht ins Innere der Drïsen eindringen, sondern sich nur äusserlich auf die Drüsenmembran aufheften. Die letztere zieht als eine völlig geschlossene Haut unter dem, meist etwas kegelförmig verbreiterten Ende der Neuroidröhre hin. Der Inhalt beider communicirt also nicht. Man überzengt sich hiervon am besten durch Anwendung dünner Alkalien aufs frische Objekt. Der Inhalt der Malpighischen Gefässe wie der Neuroidröhren verflïssigt sich dann unter Quellung, während die Membranen ungelöst bleiben and in grösster Schärfe hervortreten. Niemals sieht man Inhalt der Drüse in die Neuroidröhre ibertreten oder umgekehrt, wie dies an Nervenhügeln von Insektenmuskeln zwischen Muskel- und Nerveninhalt unter gleichen Bedingungen beobachtet werden kann. Setzt man das frische Objekt einige Zeit der Einwirkung starker (etwa 30procentiger) Kalilauge aus, so lösen sich die Neuroidfasern von der Aussenfäche der Malpighisehen Gefässe glatt ab. Fügt man nun Wasser hinzu, sa quillt der Inhalt der Drüse enorm, tritt aber keineswegs an der Stelle wo der "Nerv" gesessen hatte aus; vielmehr erscheint an derselben Stelle nun die Drïsenmembran in vollster Deutlichkeit. - Bei den verhältnissmässig kolossalen Dimensionen des Objekts sind Irrungen hier durchaus unmöglich.

Die Fibrillen der Neuroidröhren endigen schon bevor sie die Aussenfläche des Malpighischen Gefässes erreichen, oder an dieser selbst. Sie hören anscheinend plötzlich auf, ohne vorher in Form, Dicke oder Verlauf noch irgend nennenswerthe Aenderungen erfahren zu haben. Speciell wurden auch hier Umbiegungen nicht 
beobachtet; ebensowenig ein Ausstrahlen längs der Oberfläche der Drüse. Zuweilen liegen noch im letzten Abschnitt der Röbre, nahe der Drüsenmembran einige kleine zellige Gebilde zwischen den Fibrillen.

Wie die abweichenden Angaben früherer Beobachter zu erklären seien, mag hier nicht untersucht werden. Ich will nur bemerken, dass die von uns untersuchten Objekte von verschiedenen Species (Gastropacha neustria, Dasychira pudibunda, Spilosoma, Euprepia, Bombyx mori u. a.) und zwar von Raupen der verschiedensten Entwickelungsstufen stammten. Es kommt mir darum nicht wahrscheinlich vor, dass die Verhältnisse bei anderen als den von uns untersuchten Formen principiell abweichend sein sollten. Der Umstand dass die beschriebenen Gebilde rein interperipherische Apparate darstellen und dabei weder am einen noch am andern Ende mit lebendiger Zellsubstanz in direkten Contakt treten, macht es am wahrscheinlichsten, dass sie - trotz ihrer so höchst auffälligen histiologischen Uebereinstimmung mit nervösen Organen - nur eine Art bindegewebiger Apparate darstellen, eine Art von Suspensorien, bestimmt um die Malpighischen Gefässe 'an der Aussenfläche des Darms zu fixiren. Aehnlich scheinen die entsprechenden Gebilde der Speicheldrüse und des Speichelbehälters bei Periplaneta aufgefasst werden zu müssen.

Die im Vorstehenden mitgetheilten Resultate mussten nur um so dringender dazu auffordern, nach Objekten zu suchen, an welchen die cardinale, physiologischerseits postalirte Thatsache des Eindringens echter Nerven in eine Drüse mit Sicherheit zu erkennen und bequem zu demonstriren war. Wir haben zu diesem Zwecke verschiedene Drïsenarten bei einer sehr grossen Anzahl der verschiedensten Arthropoden untersucht. Am meisten versprachen wir uns von den Speicheldrïsen der Wespen und Bienen, bei denen die terminalen Drïsenbläschen nur von je einer einzigen grossen kugligen Zelle gebildet werden, die wie die Beeren einer Traube mittelst dünner, oft langer Stiele dem Sammelrohr der Drïse aufsitzen. Dabei fehlt ihnen umspinnendes und interstitielles Bindegewebe fast vollständig.

Allerdings fanden wir nun auch an jedem Bläschen ein oder mehrere feine Fäserchen, die weder Tracheen waren noch Bindegewebsfasern zu sein schienen; aber es gelang uns nicht, sie im Zusammenhang mit echten Nervenstämmen zu präpariren. Auch 
waren sie von so äusserst geringer Dicke, dass über ihre nähere Verbindung mit der Drüsenzelle kaum etwas Sicheres zu ermitteln war.

Ein ausgezeichnetes Objeikt dagegen, welches hiermit der Anfmerksamkeit der Fachgenossen aufs Angelegentlichste empfohlea sei, bieten die Speicheldrüsen der Hummeln (Bombus). Die Driisenbläschen haben hier die Gestalt keulenförmiger oder kolbenförmiger Endanschwellungen der schlanken Endäste des Ausführungsrohrs der Driise. In der Länge messen sie etwa 0,2-0,4 mm, in der grössten Breite etwa 0,06-0,08 mm. Jedes Kölbchen besteht aus einer einfachen glatten strukturlosen Membran, welche immer von einem einfachen Belag grosser Drüsenzellen ausgekleidet wird. Nur ein kleines Lumen bleibt übrig in Gestalt eines von zarter Cuticula begrenzten, wellenförmig der Länge nach das Kölbchen durchziehenden, blind und meist einfach endenden, cylindrischen Canals, der sich ohne scharfe Grenze in das allmählich weiter werdende Lumen des Ausführungsgang's fortsetzt.

Die Drüsenzellen, durchschnittlich 10-15 an der Zahl, sind ziemlich isodiametrisch, sehr gross $(0,02-0,035 \mathrm{~mm})$, membranlos, ihre Grenzen im frischen Zustand nicht deutlich zu erkennen, wie ja sehr häufig bei Drüsen. Das Protoplasma ist im peripherischen Theil der Zellen, zwischen Kern und Membrana propria etwas stärker lichtbrechend, scheinbar homogener als in dem nach dem Drïsenlumen zu gelegenen Theil des Zellkörpers. Jede Zelle enthält einen $0,01-0,015 \mathrm{~mm}$ grossen bläschenförmigen Kern, der fast genau central liegt.

Für den vorliegenden Zweck werthvoll ist die fast völlig freie Lage der Drïsenkölbchen. Von interstitiellem Bindegewebe ist kaum eine Spur vorhanden. Auch der Tracheenreichthum ist mässig, obschon an jedes Kölbchen ein oder mehrere feine Endzweige sich begeben. Unter diesen Umständen ist es leicht, den grösseren Nerv zu finden und zu verfolgen, welcher sich in der Driise zwischen den Kölbchen verzweigt und auf diesem Wege sich in eine, der Zahl der Drïsenkölbchen wie es scheint, wenigstens gleiche, Zahl von Endzweigen auflöst. Jedes Drüsenkölbchen scheint nur einen, höchstens zwei Nervenzweige zu erhalten. Die Endzweige, die Cylinderform und dabei oft noch sebr erhebliche Länge $(0,05-0,2 \mathrm{~mm})$ besitzen, messen durchschnittlich $0,005-$ 
$0,01 \mathrm{~mm}$ in der Dicke. Sie endigen meist im mittleren Drittel des Kölbchens, seltener an der Spitze, und zwar durchbohren sie die Wand der Drüse. Ihre ziemlich dicke Scheide verschmilzt mit der Membrana propria, die Nervenfibrillen treten mit den Drüsenzellen in Contakt.

Es ist leicht, sich völlig überzengende Profilbilder dieses Eintritts zu verschaffen. Sehon das frische Objekt giebt gute Ansichten. Zusatz dünner Kalilange lässt dann die Continuität der Scheiden und Lumina von Drüse und Nerv ganz besonders handgreiflich hervortreten.

Besondere Strukturverhältnisse bietet der Nervenendzweig beim Eintrittin die Drüse nicht dar. Erbehält sogar bis zum Durchtritt durch die Membran den gleichen Querschnitt. Auch nach dem Durchtritt durch die Drüsenmembran lassen sich keine besonderen Bildungen an ihm erkennen: die Fïbrillen treten geradeswegs zum Zellprotoplasma. Ob sie sich nur zu den zunächst der Eintrittsstelle gelegenen Zellen begeben, oder innerhalb der Drüse sich noch weiter verästeln, so dass mehrere eventuell jede der Zellen des nämlichen Kölbchens ein eigenes Nervenende erhält, liess sich nicht entscheiden, da es nicht gelingen wollte, ein Mittel zu finden, welches die Nervenfibrillen vom anliegenden Drüsenprotoplasma sicher zu unterscheiden gestattet hätte.

Die allgemeine Bemerkung möge aber hier einen Platz finden, dass physiologischerseits einstweilen durchaus kein Grund für die Annahme vorliegt, dass jede einzelne Zelle eines Drïsenkölbchens ihren eigenen Nerv bekomme. Da die Zellen nicht durch Membranen von einander getrennt, sondern in so innigem Contakt mit einander sind, dass sie im Leben vielmehr eine continuirliche Protoplasmamasse als eine Mosaik selbstständiger Zellindividuen zu bilden scheinen, so genügt es für das Zustandekommen einer Uebertragung des Nervenreizes auf alle in einem Kölbchen golegenen Zellen. vollkommen, wenn nur eine einzige Zelle direkt mit dem Nerv zusammenhängt. Es ist hier ganz dasselbe Verhältniss, auf welches ich zuerst beim Ureter, dann bei Flimmerepithelien, beim Herzmuskel und bei glatten Muskeln überhaupt, aufmerksam gemacht habe und das auch bei der Nervenleitung selbst in Anwendung kommt: Mittheilung der Erregung durch Zellcontakt.

Eine Frage, die im Auge behalten zu werden verdient, ist, ob auch eine Leitung der Erregung von einem Drüsenkölbchen zum 
andern durch Vermittlung der Zellen der die Kölbchen verbindenden Ausfithrungsgänge stattfinden könne. Im vorliegenden Falle möchte dies wegen der sehr abweichenden Eigenschaften der Epithelzellen der Ausfiuhrungsgänge und des Umstands wegen, dass jedes Kölbchen doch einen eigenen Nerven erhält, nicht wahrscheinlich sein. Aber es wäre doch möglich, dass bei anderen Drüsen auf dem angedenteten Wege eine reizleitende Verbindung und damit eine gleichsam nervöse Verkettung zwischen einer grösseren Anzahl von Drüsenbläschen oder -schläuchen hergestellt würde. Hier würden dann für grössere Aggregate der letzteren, eventuell für die ganze Drïse nur eine oder wenige Nervenendigungen genügen. Sollte hierin vielleicht der Schlüssel zur Erklärung so vieler erfolgloser Bemühungen, Nervenendigungen in Drüsen zu finden, und damit ein Weg gegeben sein zur Lösung der auf diesem Gebiet der mikroskopischen Anatomie bestehenden Differenzen?

Agriculturchemisches Laboratorium der landwirthsch. Versuchsstation in Kiel.

\section{Ueber die durch Einwirkung von Kaliumhydrat auf Traubenzucker entstehende reducirende Substanz.}

Von

Dr. A. Tmmerling und Dr. O. Loges.

Vor Kurzem haben Prof. Worm Müller, und J. Hagen in dieser Zeitschrift ${ }^{1}$ ) an die schon von $K$ iih ne beobachtete interessante Erscheinung erinnert, dass der Traubenzucker, mit Kali kurze Zeit erwärmt und wieder abgekühlt, die Fähigkeit annimmt, Kupferoxyd schon in der Kälte zu reduciren. Es entstehen biernach

1) Bd. XXII. p. 391 . 